\title{
Streptavidin Affinity Grids for Single-particle Cryo-EM
}

\author{
Robert M. Glaeser ${ }^{1 *}$ and Bong-Gyoon $\operatorname{Han}^{1}$ \\ 1. MBIB Division, Lawrence Berkeley National Lab, Berkeley, CA, USA. \\ * Corresponding author: rmglaeser@1bl.gov
}

Unless biological macromolecules are immobilized tightly onto a thin support film before excess sample is removed, contact between them and the air-water interface is almost unavoidable prior to vitrification [1]. However, immobilization onto support films, such as evaporated carbon or graphene oxide, can itself damage macromolecular structures. It thus is important that the interactions used to immobilize particles should not only be tight but also structure-friendly. Further, it is important that the support films should contribute as little structural noise to the image as is possible, in order to not interfere with particle alignment and Euler angle assignment during data processing. As was pointed out by [2], monolayer crystals of streptavidin (SA) have many features that are desired in an affinity support film. Biotinylated particles are bound as tightly as if by a covalent bond; the biotin-streptavidin interaction is widely used in biochemistry and cell biology, and it appears to not disrupt macromolecular function or structure; and the fixed-pattern structure of the SA crystal is easily removed from images by Fourier filtration. In addition, we have pointed out that random biotinylation of one or two lysine residues on the surface of a particle is generally expected to overcome the problem of preferential particle orientations [3].

We here address the remaining problem of how to make SA affinity grids be readily available to the wider community. To that end, we have previously developed a version of such grids that can be prepared in advance and stored until needed [4]. To achieve this, SA monolayer crystals are first grown on biotinylated lipid monolayers that span the open holes of a holey-carbon support film. As is illustrated schematically in Figure 1, these crystals are then embedded in trehalose to allow being airdried, after which a thin layer of carbon is evaporated onto the tails of the lipid monolayer to provide additional mechanical strength. The trehalose embedment is easily washed off, allowing one to apply a biotinylated sample, and then either negatively stained grids or cryo-EM grids can be prepared in the usual way. Although a few laboratories have begun to independently make and use such grids, the step of making SA affinity grids is still time-consuming, and this added step remains a barrier for most. Thus, in order to scale up the rate at which such grids can be prepared, by a factor of 10 or more, we now are experimenting with ways to hold multiple grids through each step of the process, beginning with transfer of the lipid monolayer to holey-carbon grids through the evaporation of carbon onto the lipid tails.

The use of affinity grids does not address another problem in single-particle cryo-EM, namely the need to control the thicknesses of the thin aqueous films that remain after removal of excess sample material. The results obtained by blotting with filter paper, when using the SA affinity grids, are neither better nor worse in this regard than for other grids. As a result, care still must be taken to confirm that dewetting of immobilized particles did not occur in areas chosen for data collection. We have reported that the resolution of ribosome-particle reconstructions, Figure 2, is correlated with the resolution of the underlying SA lattice, an effect that might be due to local dewetting in areas were the sample had gotten too thin before vitrification [5]. It is thus a benefit that the values of resolution to which SA-crystal Bragg spots are seen in the FFT of images, during data collection, can be used to get feedback in real time, whether full hydration of samples has been retained in areas targeted for data collection [6]. 


\section{References:}

[1] KA Taylor and RM Glaeser, Journal of Structural Biology 163 (2008), p. 214.

[2] LG Wang, P Ounjai and FJ Sigworth, Journal of Structural Biology 164 (2008), p. 190.

[3] B-G Han et al., Journal of Structural Biology 180 (2012), p. 249.

[4] B-G Han et al., Journal of Structural Biology 195 (2016), p. 238.

[5] B-G Han et al., Journal of Structural Biology 200 (2017), p. 307.

[6] This work was supported in part by NIH grant GM051487. We especially thank Zoe Watson and Prof. Jamie Cate, who have been key collaborators in much of this work.
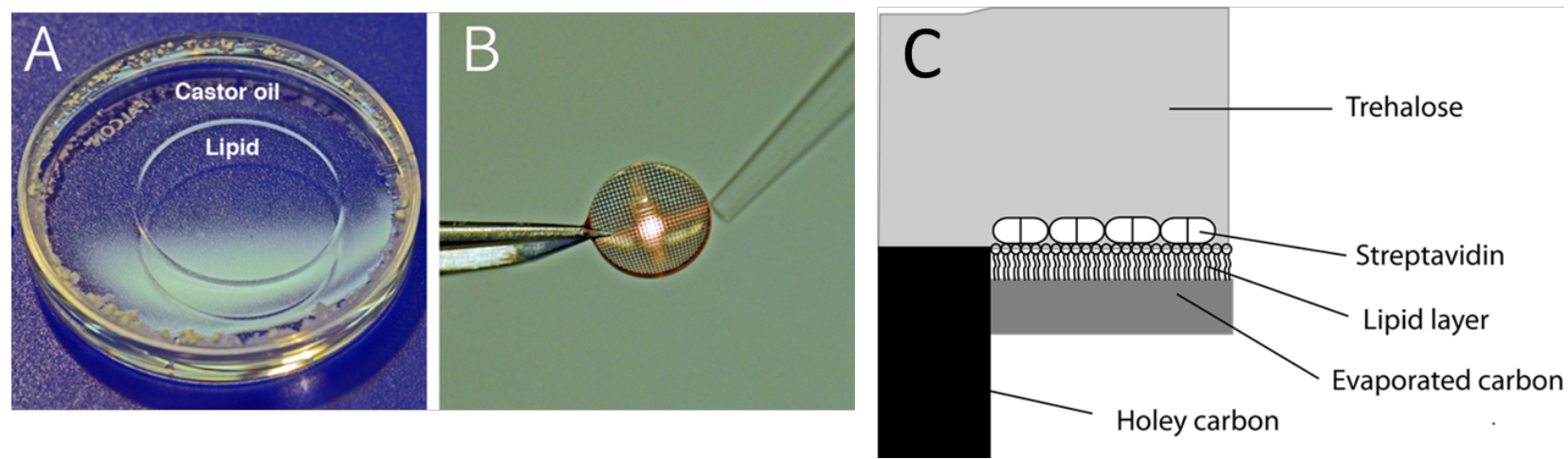

Figure 1. Illustration of how long shelf-life, SA affinity grids are made. (A) A monolayer of biotinylated lipid is spread at a controlled surface tension and transferred to holey-carbon film on an EM grid by the Langmuir-Schaefer method. (B) Streptavidin is injected into the adhering drop of buffer, and monolayer crystals are formed within 30 minutes. (C) After washing with buffer containing trehalose, the excess is wicked off and the remainder is allowed to dry. This not only allows indefinite storage of grids for future use, but in addition a thin layer of carbon can be deposited in a vacuum evaporator to provide additional mechanical strength.

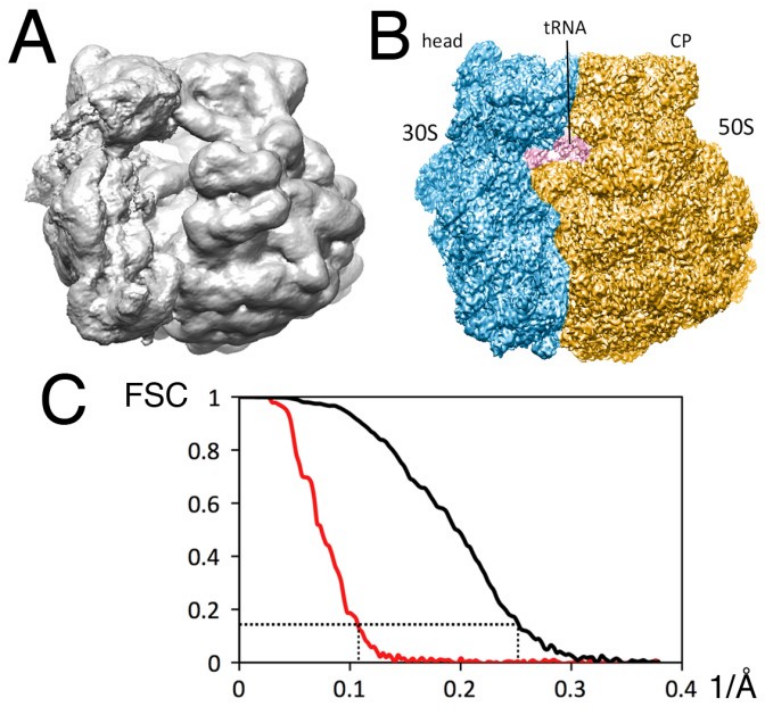

Figure 2. Comparison of the density maps of ribosome particles that where obtained from two kinds of areas of the SA affinity grids. (A) Only a lowresolution map is obtained for ribosomes if the resolution of the underlying SA lattice also extends to only low resolution. (B) A high resolution map of ribosomes is obtained, however, when the resolution of the underlying SA lattice extends to high resolution. (C) The Fourier shell correlation curves are shown for the two ribosome density maps in (A, red) and (B, black), respectively. 